EPJ Web of Conferences 81, 04005 (2014)

DOI: $10.1051 /$ epjconf/20148104005

(C) Owned by the authors, published by EDP Sciences, 2014

\title{
Exclusive production in CMS
}

\author{
Gustavo Gil da Silveira ${ }^{1}$, a for the CMS Collaboration \\ ${ }^{1}$ Centre for Cosmology, Particle Physics and Phenomenology (CP3), Université Catholique de Louvain UCL, \\ Belgium
}

\begin{abstract}
We discuss the CMS capabilities of measuring vector mesons in $p p$ and heavyion collisions based on the recent results of the exclusive dilepton and diboson production. Studies evaluating the detection of exclusive $J / \psi$ and $\Upsilon$ vector meson photoproduction in the dimuon decay channel are presented. As a result, we report the measurements performed with the CMS detector that are in the invariant mass range relevant for future studies of exclusive vector meson photoproduction in CMS.
\end{abstract}

\section{Introduction}

The CMS detector has been studying different production channels of exclusive and diffractive processes at high energies, in some cases widening the previous results obtained by other experiments. Recent studies involving photon interactions have presented outstanding results, e.g., the measurement of $W$ pairs in the electron-muon decay channel [1]. Moreover, the diffractive production of diphotons by means of pomeron exchange in the $t$-channel has reported an upper limit to the production cross-section and is in agreement with theoretical predictions [2]. In addition, the production of vector mesons are also possible in collisions involving heavy ions beams, which provides a signal enhancement due to the large number of protons in the nucleus, increasing the photon flux [3]. The recent results reported by CMS are relevant for future measurements regarding the photoproduction of vector mesons.

\section{Capabilities for meson photo-production in CMS}

Presenting an excellent efficiency in detecting muons [4], the CMS detector takes advantage of its capability to measure different production processes in the dimuon decay channel. The CMS detector can explore the region of small $\ell^{+} \ell^{-}$invariant masses where the measurement of exclusive $J / \psi$ and $\Upsilon$ photoproduction in $p p$ collisions are possible. This topics have been recently investigated in theoretical works [5-7].

A study has been performed to evaluate the capability of CMS in detecting such processes in $p p$ collisions [8]. For the exclusive photoproduction, a double muon trigger is used to collect dimuon events, requiring a vertex with exactly two muons and no additional tracks. As a exclusivity criteria, the pairs are required to have $\Delta \phi\left(\mu^{+} \mu^{-}\right)>0.9$ and $\Delta p_{T}\left(\mu^{+} \mu^{-}\right)<1.5$. Figure 1 (left) shows the event display of a candidate for the exclusive $J / \psi$ photoproduction, having an energy deposit at the hadronic

a. e-mail: gustavo.silveira@cern.ch 

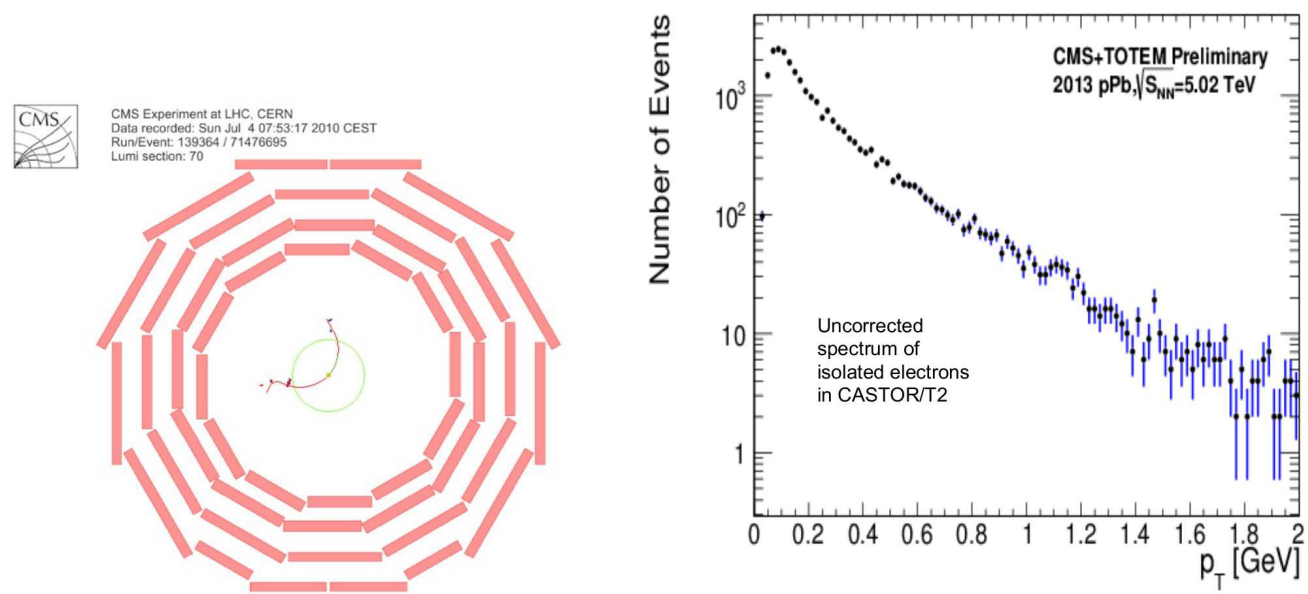

Figure 1. (left panel) Event display of a $J / \psi \rightarrow \mu^{+} \mu^{-}$candidate. A trigger selecting dimuon events is applied together with a requirement of exactly two muons in the vertex with no additional tracks. (right panel) Transverse momentum distribution of $e^{ \pm}$obtained in a detector performance study for a combined CMS-TOTEM data-taking of $p \mathrm{~Pb}$ collisions at $5.02 \mathrm{TeV}$, with an average $\eta$ of 6.0 .

and electromagnetic calorimeters greater than 4.0 and $2.5 \mathrm{GeV}$, respectively. As a result, the CMS detector allows the search for vector meson produced in exclusive events, which is corroborated by recent results in photon physics.

In the case of $p \mathrm{~Pb}$ and $\mathrm{PbPb}$ collisions, a parallel data-taking can be performed with the TOTEM experiment in order to increase the CMS coverage. The $e^{+} e^{-}$production has been studied in $p \mathrm{~Pb}$ collisions at 5.02 $\mathrm{TeV}$ to evaluate the performance of the detector [9], with the $p_{T}\left(e^{+} e^{-}\right)$distribution shown in Fig. 1 (right). Since the dilepton final state is similar to the decay of a vector meson, the CMS detector has the features to detect exclusive vector meson photoproduction in heavy ions collisions at high energies also in the very forward phase space.

\section{CMS measurements of photon-photon processes}

We report the measurements of two-photon processes performed with the CMS detector using the data collected at $\sqrt{s}=7 \mathrm{TeV}$ during 2010 and 2011. The data in these measurements concerns two regions of the invariant mass: (i) region of $M\left(\ell^{+} \ell^{-}\right)<20 \mathrm{GeV}$ for $\gamma \gamma \rightarrow e^{+} e^{-}$in $36 \mathrm{pb}^{-1}$ [2] and $\gamma \gamma \rightarrow \mu^{+} \mu^{-}$in $40 \mathrm{pb}^{-1}$ [10], and (ii) region of $M\left(\ell^{+} \ell^{-}\right)>20 \mathrm{GeV}$ for $\gamma \gamma \rightarrow \mu^{+} \mu^{-}$in $5.24 \mathrm{fb}^{-1}$ and $\gamma \gamma \rightarrow W^{+} W^{-} \rightarrow e^{ \pm} v \mu^{\mp} \bar{v}$ in $5.05 \mathrm{fb}^{-1}[1]$.

The measurement of dimuons with invariant mass greater than $20 \mathrm{GeV}$ has been used to validate the selection criteria applied in the study of $\gamma \gamma \rightarrow W^{+} W^{-}$. As shown in Fig. 2, the $p_{T}\left(\mu^{+} \mu^{-}\right)$distribution, obtained with no additional tracks in the $\left(\mu^{+} \mu^{-}\right)$vertex has good agreement between the data and the theoretical predictions. The study of the exclusive two-photon production of $W^{+} W^{-}$has found two events passing the selection requirements in the region $p_{T}\left(e^{ \pm} \mu^{\mp}\right)>30 \mathrm{GeV}, M\left(e^{ \pm} \mu^{\mp}\right)>20 \mathrm{GeV}$ and $\left|\eta\left(e^{ \pm} \mu^{\mp}\right)\right|<2.4$. Figure 2 present the acoplanarity distribution with the two events, being in agreement with the standard model expectation of $2.2 \pm 0.4$ signal events. As for the exclusivity selection, the lepton pairs are required to be within $1-|\Delta \phi(e \mu) / \pi|<0.1$ and $\Delta p_{T}\left(\mu^{ \pm} e^{\mp}\right)<1.0 \mathrm{GeV}$ and with no additional tracks from the $\mu^{ \pm} e^{\mp}$ vertex. A recent work has discussed sub-leading processes that contribute to this process [11]. 

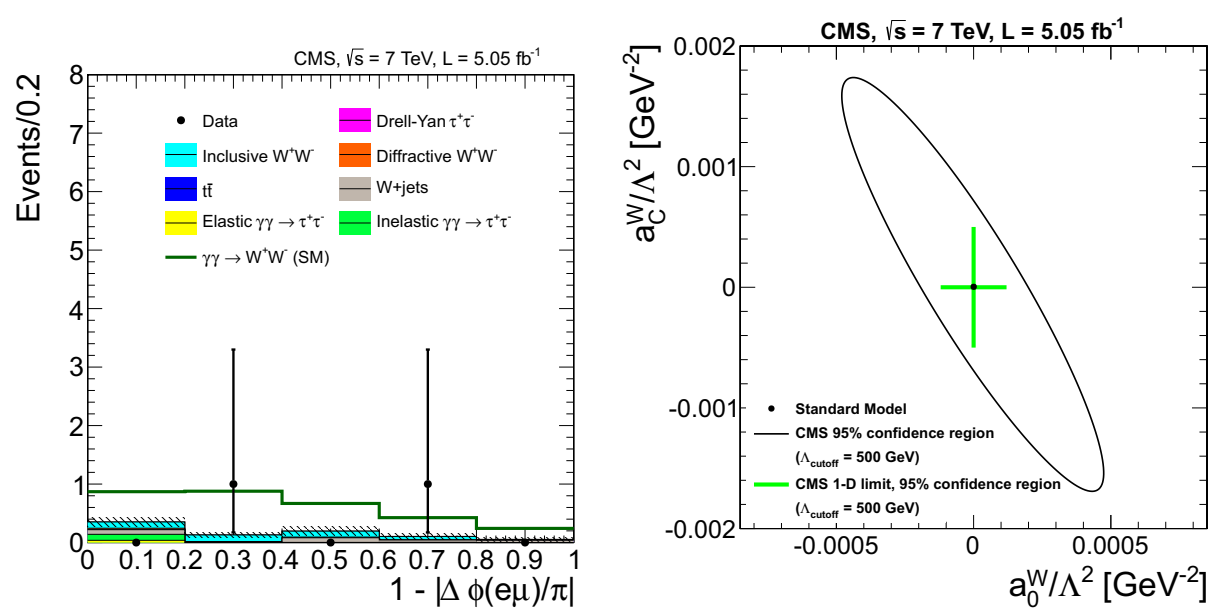

Figure 2. (left panel) The $\mu^{ \pm} e^{\mp}$ acoplanarity for events in the signal region with no additional tracks on the $\mu^{ \pm} e^{\mp}$ vertex and $p_{T}\left(\mu^{ \pm} e^{\mp}\right)>30 \mathrm{GeV}$. (right panel) Ellipse showing the values of the anomalous coupling parameters $a_{0, C}^{W} / \Lambda^{2}$ that have been found at $95 \%$ confidence level, taking $\Lambda=500 \mathrm{GeV}$.

As an extension of this study, anomalous couplings for the $\gamma \gamma \rightarrow W^{+} W^{-}$vertex are also considered. No additional events are observed in comparison to the standard model expectation. Then, new limits for the anomalous coupling parameters are obtained and are shown in Fig 2. These results are about two orders of magnitude more stringent than those obtained in LEP [12-15] and 20 times more stringent obtained by the Tevatron [16].

Although the measurement of the two-photon production of muon pairs at high masses provides the necessary understanding to report the first evidence of a $\gamma \gamma \rightarrow W^{+} W^{-}$events, the results of the exclusive dilepton production at low masses are especially important as they are a possible background events for the study of vector meson photoproduction in CMS.

The measurement of the two-photon production of $e^{+} e^{-}$at low masses has been performed taking into account the kinematic regions of $E_{T}(e)>5.5 \mathrm{GeV}$ and $|\eta(e)|<2.5$. Including the contribution of proton dissociation, 17 events have been found, which is in agreement with the standard model expectation. Figure 3 shows the $p_{T}\left(e^{+} e^{-}\right)$distribution in comparison to the predictions for the three contributions for the $\gamma \gamma \rightarrow e^{+} e^{-}$process. In the case of $\mu^{+} \mu^{-}$, cuts are applied on single muons, $p_{T}(\mu)>4 \mathrm{GeV}$ and $|\eta(\mu)|<2.1$, and muon pairs, $m\left(\mu^{+} \mu^{-}\right)>11.5 \mathrm{GeV}, 1-\left|\Delta \phi\left(\mu^{+} \mu^{-}\right) / \pi\right|<0.1$ and $\left|\Delta p_{T}\left(\mu^{+} \mu^{-}\right)\right|<1.0$. In Fig. 3 the $p_{T}\left(\mu^{+} \mu^{-}\right)$distribution is presented including the predictions for the $\gamma \gamma \rightarrow \mu^{+} \mu^{-}$process and the background events from the Drell-Yan process. As expected, a good agreement is observed between the data and theory.

\section{Conclusion}

Measurements performed with the CMS detector during the data-taking periods of 2010 and 2011 have shown promising results for the physics of two-photon processes, with remarkable agreement with the standard model expectations. These results present the capability of the CMS detector in measuring lepton pairs in both low- and high-mass regions, being the former a region for the photoproduction of vector mesons. Moreover, the results present a promising scenario for future investigations 

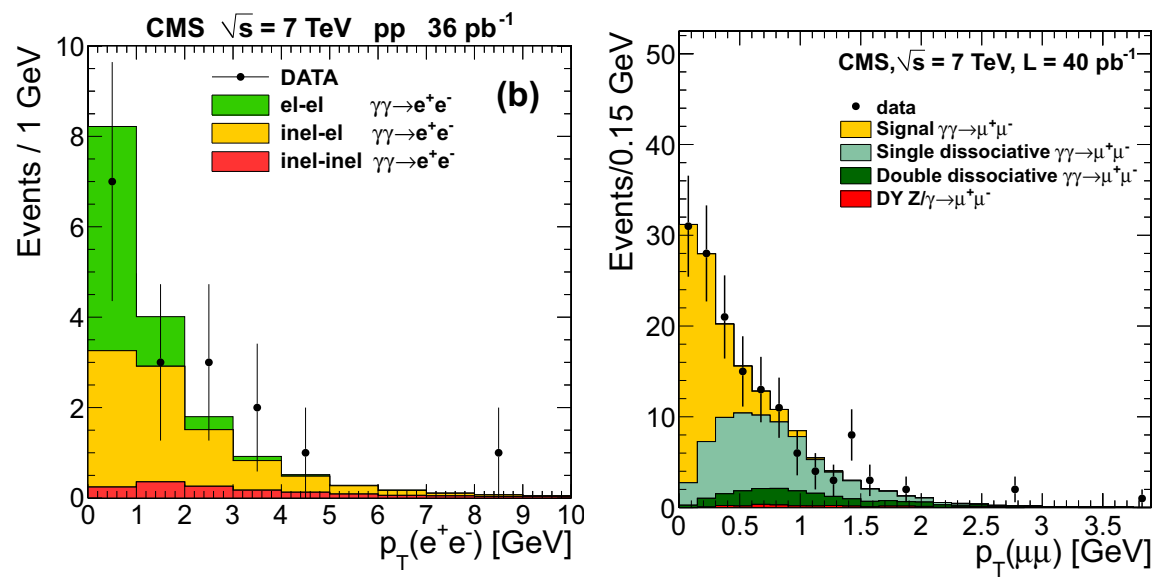

Figure 3. Transverse momentuum distributions for (left) $e^{+} e^{-}$and (right) $\mu^{+} \mu^{-}$events. Histograms show the predictions for the exclusive, single- and double-dissociative processes.

to detect vector mesons in CMS due to the same final state with lepton pairs. A combined measurement with the TOTEM experiment can provide valuable data to increase the kinematic coverage of CMS and to allow further analyses of exclusive processes.

\section{Acknowledgements}

GGS acknowledges the Brazilian Scientific agencies CNPq and Capes.

\section{References}

[1] CMS Collaboration, JHEP 07, 116 (2013).

[2] CMS Collaboration, JHEP 11, 080 (2012).

[3] A.J. Baltz et al., Phys. Rep. 458, 1 (2008).

[4] CMS Collaboration, J. Phys. G 34, 995 (2007).

[5] L. Motyka, G. Watt, Phys. Rev. D 78, 014023 (2008).

[6] V.P.B. Gonçalves, B.D. Moreira, F. Navarra, Phys. Rev. C 90, 015203 (2014).

[7] V.P.B. Gonçalves, B.D. Moreira, F. Navarra, arXiv:1408.1344 [hep-ph].

[8] CMS Collaboration, CMS-DP-2010-035 (2010).

[9] CMS Collaboration, CMS-DP-2014-014 (2014).

[10] CMS Collaboration, JHEP 01, 052 (2012).

[11] P. Lebiedowicz, R. Pasechnik, A. Szczurek, Nucl. Phys. B 867, 61 (2013).

[12] ALEPH Collaboration, Phys. Lett. B 602, 31 (2004).

[13] OPAL Collaboration, Phys. Rev. D 70, 032005 (2004).

[14] L3 Collaboration, Phys. Lett. B 527, 29 (2002).

[15] DELPHI Collaboration, Eur. Phys. J. C 31, 139 (2003).

[16] DØCollaboration, Phys. Rev. D 88, 012005 (2013). 